\title{
Persistent impairment in working memory following severe hyperglycemia in newly diagnosed type 2 diabetes
}

\author{
Joseph Cerasuolo and Anthony Izzo \\ Department of Neurology and Department of Internal Medicine, St. Vincent Hospital, Worcester, Massachusetts, USA
}

Correspondence

should be addressed

to A Izzo

Email

anthony.izzo@

stvincenthospital.com

\section{Summary}

Acute hyperglycemia has been shown to cause cognitive impairments in animal models. There is growing appreciation of the numerous effects of hyperglycemia on neuronal function as well as blood-brain barrier function. In humans, hypoglycemia is well known to cause cognitive deficits acutely, but hyperglycemia has been less well studied. We present a case of selective neurocognitive deficits in the setting of acute hyperglycemia. A 60-year-old man was admitted to the hospital for an episode of acute hyperglycemia in the setting of newly diagnosed diabetes mellitus precipitated by steroid use. He was managed with insulin therapy and discharged home, and later, presented with complaints of memory impairment. Deficits included impairment in his declarative and working memory, to the point of significant impairment in his overall functioning. The patient had no structural lesions on MRI imaging of the brain or other systemic illnesses to explain his specific deficits. We suggest that his acute hyperglycemia may have caused neurological injury, and may be responsible for our patient's memory complaints.

\section{Learning points:}

- Acute hyperglycemia has been associated with poor outcomes in several different central nervous system injuries including cerebrovascular accident and hypoxic injury.

- Hyperglycemia is responsible for accumulation of reactive oxygen species in the brain, resulting in advanced glycosylated end products and a proinflammatory response that may lead to cellular injury.

- Further research is needed to define the impact of both acute and chronic hyperglycemia on cognitive impairment and memory.

\section{Background}

The prevalence of diabetes and obesity are increasing in many different parts of the world. The major complications of diabetes are well known and are generally classified as macro- and micro-vascular complications. Treatment of the diabetes is generally aimed at reducing the risk of these chronic complications.

Acute complications of diabetes include ketoacidosis, hyperosmolar states and various metabolic derangements. Chronic hyperglycemia and diabetes have been associated with vascular dementia and an increased risk of Alzheimer disease, but no such relationship has been shown with acute episodes of severe hyperglycemia (1). Although hypoglycemia is known to cause acute alterations in mentation and consciousness, acute hyperglycemia and its effects on mental status are less well studied.

Studies done initially in rodents and more recently in the zebrafish model have shown that both hypoglycemia and hyperglycemia can impair memory (2). These deficits in zebrafish occurred after 14 days of an environment of $111 \mathrm{mM}$ glucose (equivalent to $1998 \mathrm{mg} / \mathrm{dL}$ ) and were 
found to be sustained. Previous studies in humans have shown the cognitive enhancing effects of glucose intake, but with attenuation of this effect and possible decrease in declarative memory at higher blood glucose levels (3). One study in patients with type 2 diabetes showed that not only was poor glycemic control associated with declarative memory deficits, but that ingestion of carbohydrates worsened these deficits acutely (4).

To our knowledge, there are no reported cases of persistent cognitive deficits in adult patients after an episode of acute hyperglycemia. We described the case of a patient diagnosed with new-onset type 2 diabetes, who developed persistent impairment in his working and declarative memory without any other identifiable cause.

\section{Case presentation}

A 60-year-old man was admitted to the hospital from his primary care physician's office due to altered mental status and severe hyperglycemia. He had a history of anxiety, depression and hepatitis $\mathrm{C}$ infection. On review of his medical records, he had an elevated venous blood glucose level of $121 \mathrm{mg} / \mathrm{dL}$ two years prior, but no history of diabetes. His medications on admission included venlafaxine, risperidone, carbamazepine, clonazepam, cogentin and zolpidem.

One week earlier, he had visited an urgent care center for shortness of breath and a cough, for which he was given a course of azithromycin and a five-day course of prednisone $40 \mathrm{mg}$ daily. His chest $\mathrm{X}$ ray was clear and he had no other systemic signs of infection. Over the next 2-3 days, he became altered. His spouse described excessive sleepiness, thirst and speech that was incoherent. Prior to this episode, the patient had an average level estimated pre-morbid intellect; he had received a high school diploma and was able to work successfully in both construction and the hotel industry.

The patient was noted to have severe hyperglycemia with a blood glucose of $904 \mathrm{mg} / \mathrm{dL}$ along with acute kidney injury. His basic metabolic panel showed a sodium of $120 \mathrm{mEq} / \mathrm{L}$, potassium of $5.6 \mathrm{mEq} / \mathrm{L}$, chloride of $83 \mathrm{mEq} / \mathrm{L}$, bicarbonate of $21 \mathrm{mEq} / \mathrm{L}$, BUN of $29 \mathrm{mg} / \mathrm{dL}$ and creatinine of $1.86 \mathrm{mg} / \mathrm{dL}$. His HbA1C measured on admission was $9.9 \%$. His lactate was elevated to $3.8 \mathrm{mg} / \mathrm{dL}$, but he had no systemic signs of infection. Repeat chest $X$ ray again showed no infiltrates, and no other source of infection was identified. Due to his persistent confusion in the emergency room, a CT scan without contrast of the head was done which did not show any infarcts, but did show scattered tiny hypodensities in the subcortical and deep white matter suggesting chronic small-vessel disease.

The patient was given 8 units of subcutaneous regular insulin and received a total of $5 \mathrm{~L}$ normal saline. Over the next $12 \mathrm{~h}$, his venous blood glucose had decreased to $468 \mathrm{mg} / \mathrm{dL}$, and he was started on a basal bolus insulin regimen with 20 units of insulin glargine and 6 units of nutritional insulin lispro. The patient was admitted to the hospital and his confusion slowly improved. He was answering questions appropriately and was able to follow diabetic education. By hospital day two, his finger stick blood glucose levels were more controlled ranging from 238 to $284 \mathrm{mg} / \mathrm{dL}$, and before discharge on hospital day three were controlled ranging from 162 to $244 \mathrm{mg} / \mathrm{dL}$ without any episodes of hypoglycemia. He was discharged with an insulin regimen of 24 units of insulin glargine and 8 units of nutritional insulin lispro.

On review of the patient's 4- and 8-week follow-up appointments with his endocrinologist, his blood glucose levels ranged from 80 to $120 \mathrm{mg} / \mathrm{dL}$. He was started on metformin and subsequently his insulin regimen adjusted to 20 units of insulin glargine daily and 6 units of nutritional insulin lispro without any hypoglycemic events. He was noted to have complaints regarding his memory since discharge from the hospital which did not improve, and so was referred for neurological evaluation.

\section{Investigation}

The patient's complaints were referable to short-term memory deficits. His spouse described him asking the same question repeatedly every couple of hours and forgetting what he was doing mid task. He was started on occupational therapy, but did not improve over 2-3 months. Examination showed an obese but otherwise healthy gentleman, full motor strength throughout, normal sensation, intact cranial nerves, normal reflexes and normal cerebellar testing. He had a mildly ataxic gait, which was a new finding. The patient had deficits in his working memory, scoring a $17 / 30$ on a Montreal Cognitive Assessment with poor performance in memory, attention and delayed recall (5). An MRI of the brain was done, which showed generalized volume loss and chronic microangiopathic changes, but otherwise no abnormalities.

\section{Treatment}

After excluding any structural abnormality in the brain or any further metabolic derangements, the patient was continued on cognitive rehabilitation. 


\section{Outcome and follow up}

The patient was seen in the neurology clinic about four months after his initial hospitalization without any significant improvement in his memory impairment. He had continued follow-up with his endocrinologist, which showed excellent glycemic control without any episodes of hypoglycemia.

\section{Discussion}

We describe a patient with acute onset of confusion in the setting of severe hyperglycemia. He was thought to be previously prediabetic due to atypical antipsychotics use, obesity and chronic hepatitis $\mathrm{C}$ infection, with his acute hyperglycemia triggered by steroid use.

The patient had no focal neurological deficits, and so was initially thought to have encephalopathy secondary to his metabolic derangements, with possible etiologies being acute hyperglycemic, hyperosmolar state and volume depletion or hyponatremia. However, his sodium level was normal when corrected for his severe hyperglycemia, and his calculated serum osmolality on admission was $301 \mathrm{mosmol} / \mathrm{kg}$ suggesting he was not in a hyperosmolar state. On retrospective review of vital signs logged at least every shift on our institution's electronic medical record, the patient was never hypertensive during his hospitalization to account for his encephalopathy. Similarly, he was never hypotensive and MRI imaging done as an outpatient showed no areas of infarct, as well as no evidence of injury or sclerosis of the hippocampus. There were no episodes of hypoxia before or during his admission in the hospital. While he recovered from his acute illness, even after several months, he was left with a prominent impairment in his working memory to the point that it impaired his function.

Our patient's blood glucose levels were slowly corrected over the course of 3 days. Although he was insulin naive, he did very well as an outpatient and had no recorded hypoglycemic events, nor could he recall any symptoms of the same, suggesting that hypoglycemia was not producing his memory complaints. Importantly, the patient did not have any changes in his medications besides his diabetes medications. Although prednisone use can cause mental status changes, he was given a relatively modest dose and a very short course, which would not explain his prolonged memory deficits.

A confounding factor in our patient's history included his prior psychiatric medications. Specifically, anticholinergic medications are well known to cause cognitive impairment (6). In fact, animal studies also seem to suggest alterations in central nervous system acetylcholine levels as possible mechanisms for memory impairment in hyperglycemia (2). In addition, zolpidem and other benzodiazepine agonists have been implicated in memory impairment (7). However, no changes were made to our patient's home medications, and in fact, they were all continued after admission to the hospital. Impairment in memory secondary to chronic use of these medications is unlikely to present as abrupt onset of new neurological symptoms, suggesting to us that the patient's acute illness is more likely responsible for his memory impairment.

Temporally, the patient's decline correlated with his severe hyperglycemic episode. We propose that the severe hyperglycemic state may have caused specific injury to the hippocampus and possibly cerebellum. Our patient had no other identifiable factors to explain his memory impairment, including any structural abnormalities on his MRI, drug or alcohol use or new medications. While metabolic encephalopathy seemed likely, his deficits were restricted to declarative memory and did not show any improvement over time after correction of his acute hyperglycemia.

The impact of hyperglycemia on brain function is not fully understood. A clear association between diabetes and dementia exists and was originally thought to be due to vascular disease. However, diabetes significantly increases the risk of developing Alzheimer type dementia, so it is likely mechanisms other than micro- and macro-vascular effects contribute (1). In fact, the deleterious effects of hyperglycemia have been described extensively and are well known in the setting of CNS injuries including stroke and hypoxic injury. He did not, however, suffer an ischemic infarct to explain his symptoms, nor would a slow, progressive disease like Alzheimer's explain the acuity of the patient's cognitive change.

Hyperglycemia is known to increase reactive oxygen species in the CNS, which is thought to be one mechanism for the macro- and micro-vascular complications of diabetes (8). This increase in reactive oxygen species is thought to be mediated by a variety of effects, including advanced glycosylated end products, induction of proinflammatory mediators in the CNS and other alterations in cellular metabolism. In addition, there is a growing body of evidence that the blood-brain barrier is significantly disrupted by hyperglycemia (9). Neuroprotective effects that tend to diminish these effects may not be effective in acute severe hyperglycemia, and we suggest this may be 
why our patient's sudden and severe hyperglycemia may have caused his observed injury. We postulate that severe hyperglycemia may injure specific areas of the brain, similar to how hypoxic brain damage tends to result in injury to predictable areas of the brain. Our case illustrates the need for further research into the mechanisms of injury and alteration in CNS function in the setting of hyperglycemia.

\section{Declaration of interest}

The authors declare that there is no conflict of interest that could be perceived as prejudicing the impartiality of the research reported.

\section{Funding}

This research did not receive any specific grant from any funding agency in the public, commercial or not-for-profit sector.

\section{Patient consent}

Informed consent was obtained from the patient for publication of this article.

\section{Author contribution statement}

The primary author was responsible for preparation of the manuscript and oversaw care of the patient in the inpatient setting. The secondary author offered revisions of the manuscript and was the primary neurology consultant to the patient.

\section{References}

1 Crane PK, Walker R, Hubbard RA, Li G, Nathan DM, Zheng H, Haneuse S, Craft S, Montine TJ, Kahn SE, et al. 2013 Glucose levels and risk of dementia. New England Journal of Medicine 369 540-548. (https://doi.org/10.1056/NEJMoa1215740)

2 Capiotti KM, De Moraes DA, Menezes FP, Kist LW, Bogo MR \& Da Silva RS 2014 Hyperglycemia induces memory impairment linked to acetylcholinesterase in zebrafish (Danio rerio). Behavioural Brain Research 274 319-325. (https://doi.org/10.1016/j.bbr.2014.08.033)

3 Parsons M \& Gold P 1992 Glucose enhancement of memory in elderly humans: an inverted-U-dose-response curve. Neurobiology of Aging 13 401-404. (https://doi.org/10.1016/0197-4580(92)90114-D)

4 Greenwood CE, Kaplan RJ, Hebblethwaite S \& Jenkins DJA 2003 Carbohydrate-induced memory impairment in adults with type 2 diabetes. Diabetes Care 26 1961-1966. (https://doi.org/10.2337/ diacare.26.7.1961)

5 Nasreddine ZS, Phillips NA, Bédirian V, Charbonneau S, Whitehead V, Collin I, Cummings JL \& Chertkow H 2005 The montreal cognitive assessment, MoCA: a brief screening tool for mild cognitive impairment. Journal of the American Geriatrics Society 53 695-699. (https://doi.org/10.1111/j.1532-5415.2005.53221.x)

6 Collamatti A, Martone AM, Poscia A, Brandi V, Celi M, Marzetti E, Cherubini A \& Landi F 2016 Anticholinergic drugs and negative outcomes in the older population: from biological plausibility to clinical evidence. Aging Clinical and Experimental Research 28 25-35. (https://doi.org/10.1007/s40520-015-0359-7)

7 Stranks EK \& Crowe SF 2014 The acute cognitive effects of zopiclone, zolpidem, zaleplon, and eszopiclone: a systematic review and metaanalysis. Journal of Clinical and Experimental Neuropsychology $\mathbf{3 6}$ 691-700. (https://doi.org/10.1080/13803395.2014.928268)

8 Giacco F \& Brownlee M 2010 Oxidative stress and diabetic complications. Circulation Research 107 1058-1070. (https://doi org/10.1161/CIRCRESAHA.110.223545)

9 Prasad S, Sajja RK, Naik P \& Cucullo L 2014 Diabetes mellitus and blood brain barrier dysfunction: an overview. Journal of Pharmacovigilence 2 125. (https://doi.org/10.4172/2329. 6887.1000125)

Received in final form 14November2017

Accepted 22November2017 